\title{
A VLSI Design Framework with Freeware CAD Tools
}

\author{
Y. K. Teh ${ }^{1}$, Student Member, IEEE, F. Mohd-Yasin ${ }^{1}$, Member, IEEE, M. B. I. Reaz ${ }^{2}$, Member, IEEE, \\ A. Kordesch ${ }^{3}$, Senior Member, IEEE \\ ${ }^{1}$ Faculty of Engineering, Multimedia University, 63100 Cyberjaya, Selangor, MALAYSIA \\ ${ }^{2}$ ECE Dept, IIUM, 53100 Kuala Lumpur, MALAYSIA \\ ${ }^{3}$ Device Modeling Group, Silterra Sdn. Bhd., Kulim, Kedah, MALAYSIA \\ E-mail: ykteh@mmu.edu.my
}

\begin{abstract}
This work presents a PC-based freeware $\mathrm{CAD}$ environment to design and tape out VLSI microelectronic circuits, starting from schematic capture all the way to a foundry compatible GDS II database. These free tools will help more Malaysian universities to set up low cost VLSI CAD laboratories and tape out circuits using Silterra's University Program. This will help grow local IC design culture and skills. FreeVLSI uses common freeware CAD tools: 5SPICE, LASI, and WinSPICE, and some custom scripts to interface between these tools. Currently FreeVLSI is able to cater to full custom design flow from schematic capture to circuit layout.
\end{abstract}

\section{INTRODUCTION}

Electronics manufacturing is one of the most important industries in Malaysia. Recently, cheap labor from other countries has prompted Malaysia to try to move up the value chain to chip design, following the Taiwan model. It is crucial to develop manpower with the IC design skills early at universities undergraduate level to achieve the objective.

In Malaysia, Silterra has offered a university program that allows university researchers to fabricate their designs at no cost, similar to [1] and [2]. The chips are designed and simulated using costly commercial design tools. We attempt one step further, by designing free tools to design chips employing Silterra technology.

The purpose of this work is to present a PCbased freeware $\mathrm{CAD}$ environment (called FreeVLSI) to design and tape out VLSI microelectronic circuits, starting from schematic capture finally producing a foundry compatible GDS II database. These free CAD tools will help Malaysian universities to set up low cost design laboratories to grow local IC design culture and skills. FreeVLSI is currently capable of accommodating full custom ASIC design flow.

Three different types of circuits (MOSFET I$\mathrm{V}$ characterizations, digital and analog) have been simulated using FreeVLSI and commercial tools. Comparisons have been made with the actual silicon data. Silterra $0.18 \mu \mathrm{m}$ CMOS SPICE models were used in all simulations.

\section{DESCRIPTION OF FRAMEWORK PROPOSED}

Figure 1 shows the freeware components proposed to accomplish FreeVLSI Full Custom design flow:

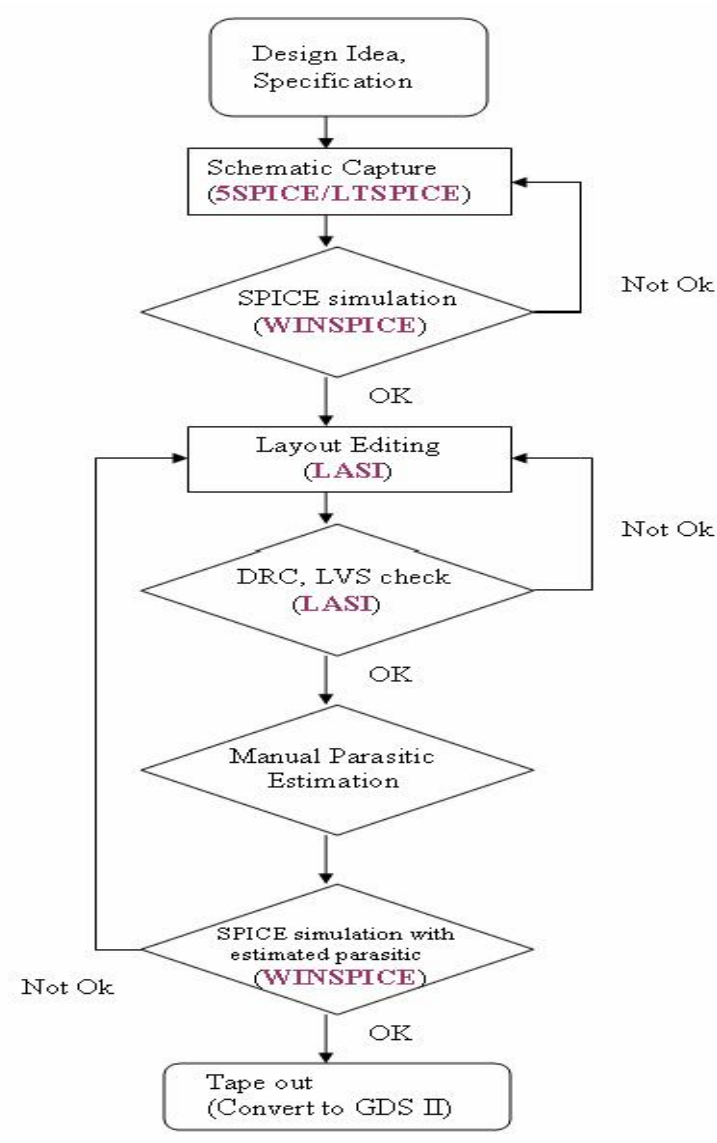

Figure 1: Full Custom Design Flow of FreeVLSI 


\section{- Schematic Capture}

Schematic capture software is used to construct circuit schematics and generate SPICE simulation net lists. Two good candidates found are 5SPICE [3] and LTSPICE [4].

Alternatively LASI which is proposed as layout editing tool could also be used to perform schematic capture and net list extraction. Nevertheless device models in extracted net lists have to be altered in order to match the device name given in foundry library. This could be done by manual editing or utilizing computer scripts.

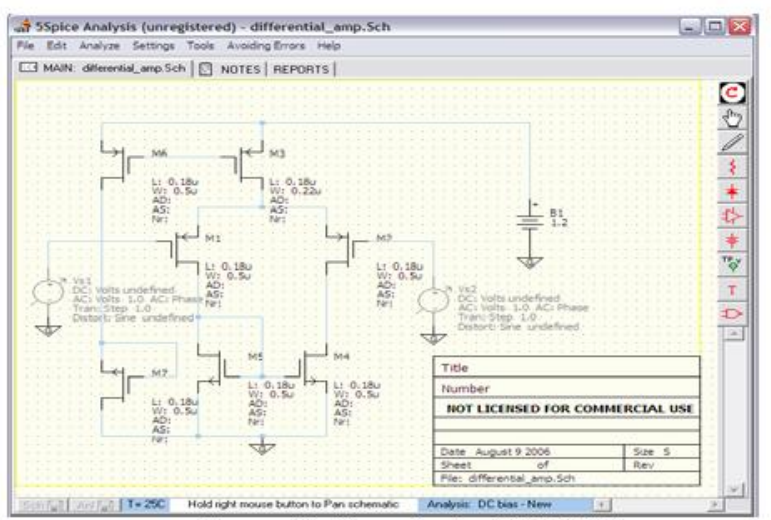

Figure 2: Snapshot of 5SPICE

\section{- SPICE Simulation}

BSIM3 is popular CMOS MOSFET device model developed by the BSIM Research Group [5] of the University of California, Berkeley. The third iteration of BSIM3, BSIM3 Version 3 (commonly abbreviated as BSIM3v3) has since been widely used by most semiconductor foundries and IC design houses world wide for device modeling and CMOS IC design.

In this framework, the target is to find freeware alternatives that are capable of simulating commercial BSIM3v3 device model libraries provided by the foundry. A good candidate discovered after testing many freeware solutions available is WINSPICE [6]. WINSPICE is a port of Berkeley's original Spice3F4 to Microsoft Windows operating system. WINSPICE is capable of taking HSPICE BSIM3V3 compatible MOSFET device models and also BJT (Gummel and Poon model) after applying software patch upgrades provided by the author. Users can use WINSPICE to simulate circuits with a mixture of MOSFET and CMOS parasitic BJT devices such as bandgap reference circuits. WINSPICE can perform both electrical simulation and graphical post processing through programming scripts.

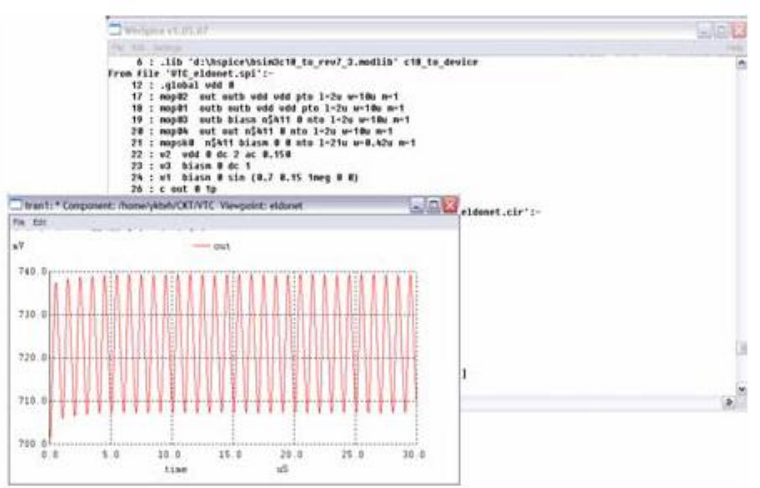

Figure 3: WinSPICE nutmeg and plot snapshot

\section{- Layout Editing}

Windows LASI [7] (Windows LAyout System for Individuals) is recommended to perform custom layout editing, Design Rule Check (DRC), macro extraction, Electrical Rule Check (ERC), Layout Versus Schematic (LVS), as well as final data codification (conversion to Gerber GDS II format for foundry fabrication). LASI was written by Dr. David Boyce. LASI runs on the Microsoft Windows system.

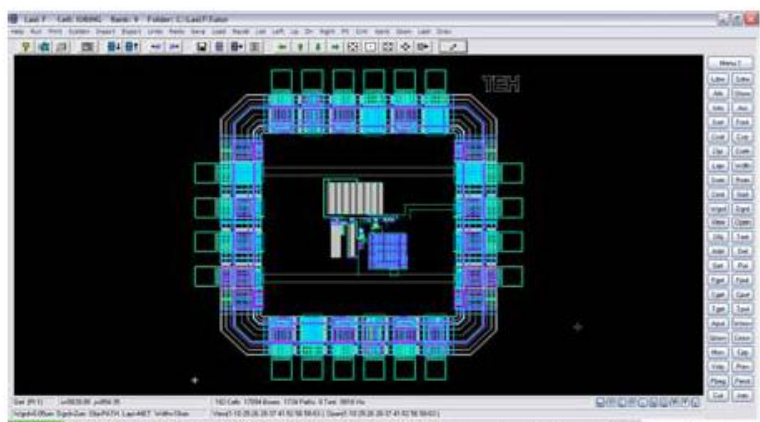

Figure 4: LASI showing fabricated bandgap design

Default set up files for LASI have been modified to match GDS layer definitions of the targeted foundry. It is worth noting that LASI is only capable of doing simple DRC check and parasitic extraction is still in development as sophisticated LASI compatible deck files need to be rewritten. In the current development of FreeVLSI, designers have to manually estimate and compute parasitic capacitance based on circuit layout and foundry electrical design rules. 


\section{DESIGN EXAMPLES}

Three different types of circuits have been simulated using FreeVLSI and commercial tools for comparison. Silterra $0.18 \mu \mathrm{m}$ CMOS technology is used in all simulations. Example circuits include MOSFET I-V characterization, Bandgap reference circuit (analog circuit) with actual silicon data, and TSPC D flip flop (digital circuit).

\section{- MOS I-V characterization}

Both NMOS and PMOS types of transistors available in Silterra's $0.18 \mu \mathrm{m}$ process are characterized using HSPICE, Eldo and WinSPICE. Saturation current of PMOS and NMOS of different $\mathrm{W} / \mathrm{L}$ ratio is extracted. Data from three SPICE simulators are plotted in Figures 5 and 6.

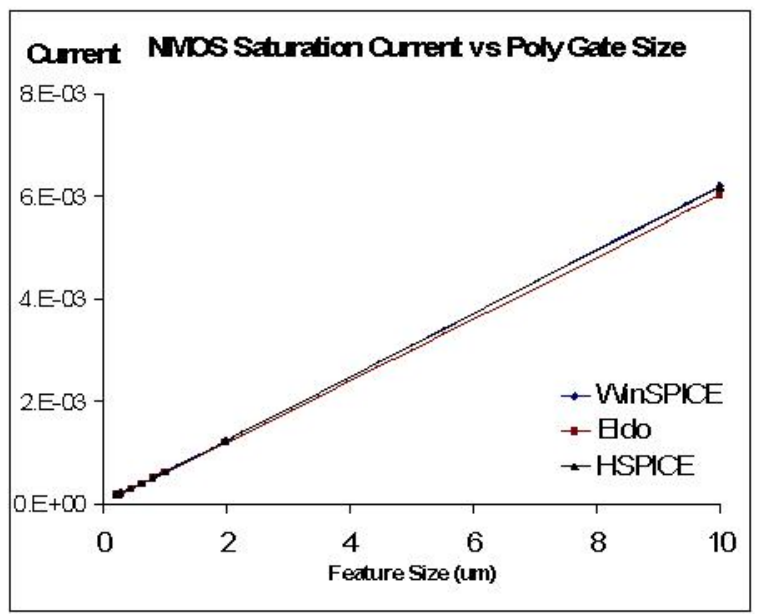

Figure 5: NMOS I $\mathrm{I}_{\mathrm{Dsa}}$ vs Poly gate size

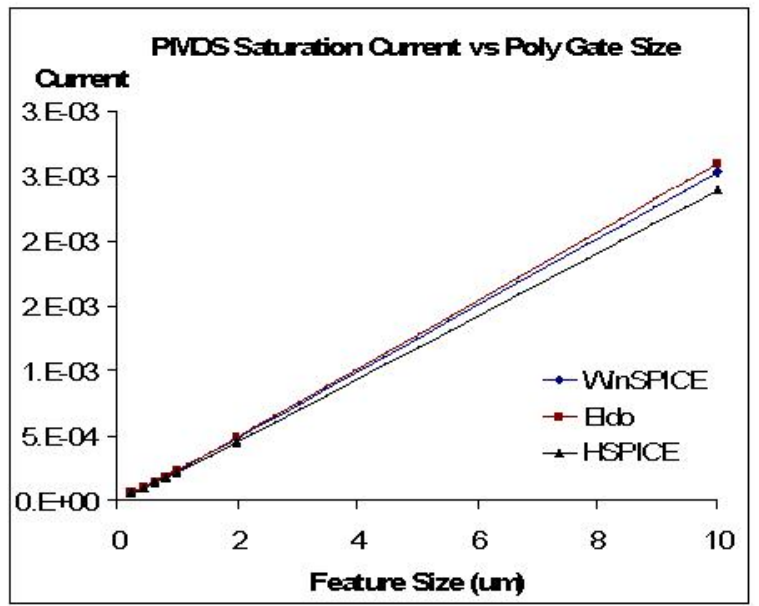

Figure 6: PMOS $I_{D \text { sat }}$ vs Poly gate size
Three simulators give close results for small feature size devices (Poly gate width less than 2um). Results are summarized below.

\begin{tabular}{|l|c|c|}
\cline { 2 - 3 } & Eldo & HSPICE \\
\hline NMOS & $3.34 \%$ & $0.88 \%$ \\
\hline PMOS & $2.07 \%$ & $5.91 \%$ \\
\hline
\end{tabular}

Figure 7: Average difference in simulation results of I-V characterization for Eldo and HSPICE compared to WinSPICE with variable $\mathrm{W}$, Fixed $\mathrm{L}=0.18 \mu \mathrm{m}$

\section{A. Analog Circuit - Bandgap Voltage Reference}

Figure 8 shows an analog circuit example, which is a low power current mode bandgap reference circuit derived from H.Banba's paper [8]. DC, AC and transient analyses are simulated on two simulators: Mentor Graphics Eldo and WINSPICE. The design has been fabricated using Silterra's $0.18 \mathrm{um}$ process and DC results are confirmed with actual silicon IV measurement using an HP4156B parameter analyzer. Simulation results obtained from both simulators are compared with actual silicon measurement data.

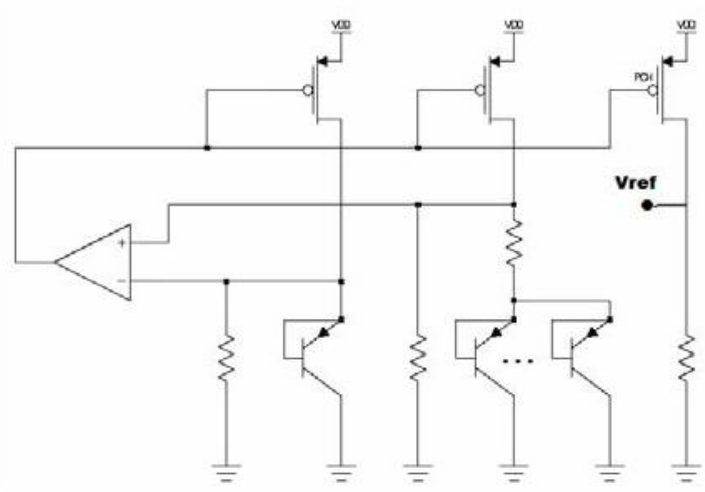

Figure 8: Bandgap reference circuit investigated

DC sweep simulation result shows less than $1 \%$ difference between commercial simulator (Eldo) and WinSPICE. Both simulators yielded good match against actual silicon results for both output voltage and current consumption as shown in Figure 9 and Figure 10. 


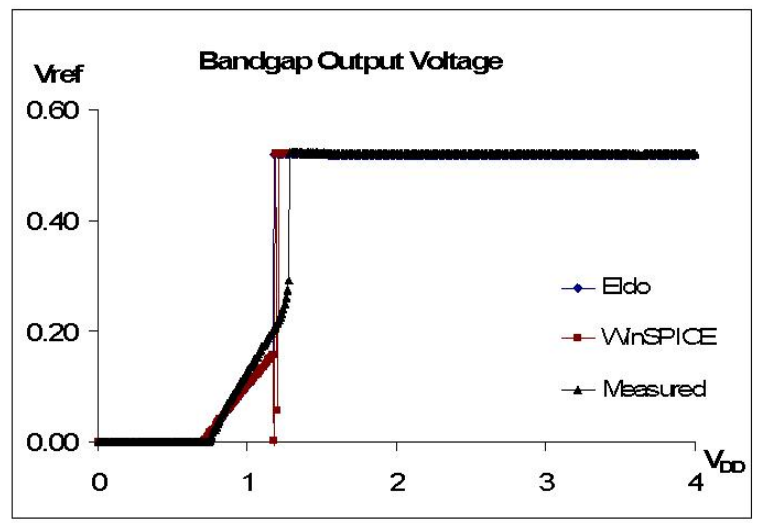

Figure 9: $\mathrm{V}_{\text {ref }}$ of Bandgap vs $\mathrm{V}_{\mathrm{DD}}$

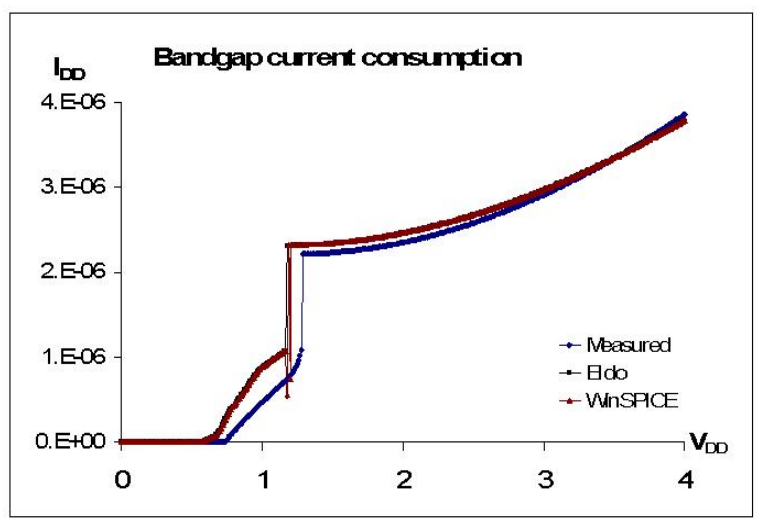

Figure 10: $\mathrm{I}_{\mathrm{DD}}$ of Bandgap vs $\mathrm{V}_{\mathrm{DD}}$

$\mathrm{AC}$ analysis, which is a common analysis performed by designers is also compared. Again both simulators give nearly identical results as shown in Figure 11 (a),(b).
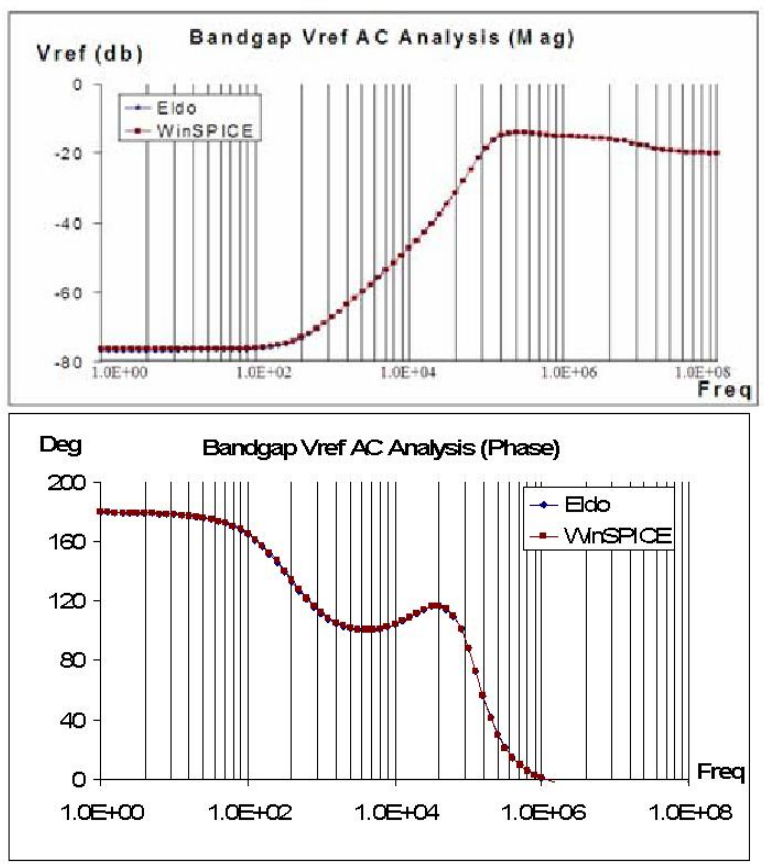

Figure 11 (a), (b): AC response of Bandgap Vref
Transient response is simulated and plotted in Figure 12. WinSPICE simulation shows some discrepancy at initial transient but overall the response tracks Eldo's output well after output voltage stabilizes.

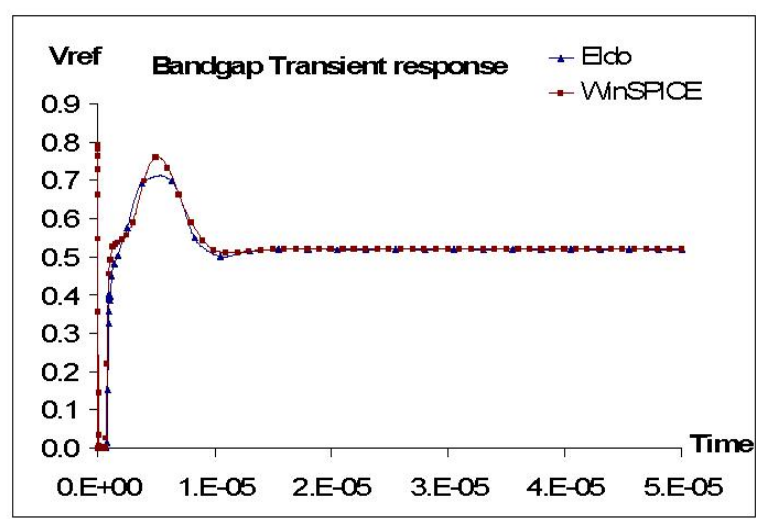

Figure 12: Transient response of Bandgap $V_{\text {ref }}$

\section{B. Digital Circuit - D Flip Flop}

Behaviour of digital circuit simulation under FreeVLSI is investigated as well. A TSPC (True Single Phase Clock) D flip flop circuit is simulated using both Eldo and WinSPICE.

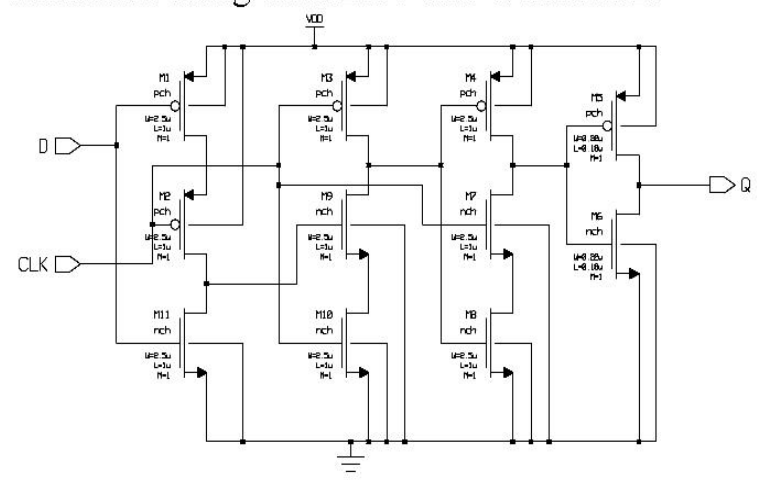

Figure 13: TSPC D Flip Flop circuit

With the same stimulus fed to CLK and D input of D flip flop, Q output of D flip flop is forced to switch at $250 \mathrm{MHz}$. Transient response of $\mathrm{Q}$ output from both simulators is plotted on Figure 14. It can be seen that simulation results yielded from Eldo and WinSPICE actually tracks closely; except the initial transient. Timing difference demonstrated by both simulators shows less than $1 \mathrm{ps}$ difference. 


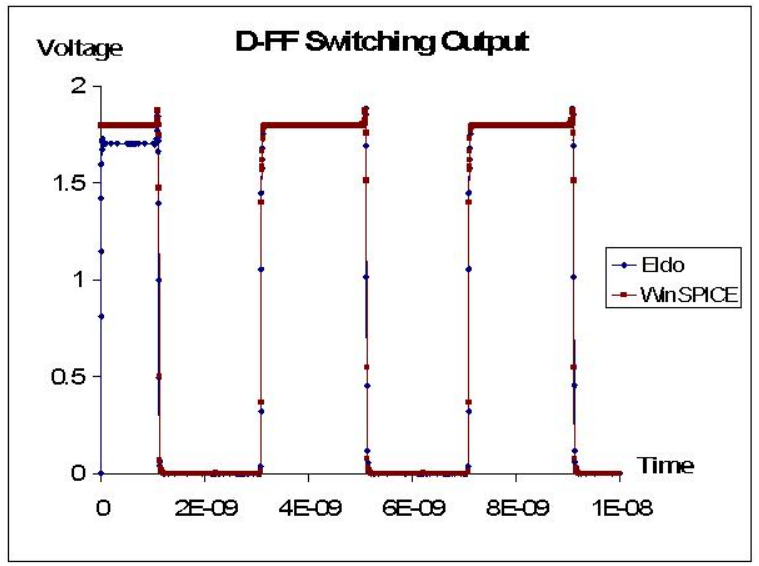

Figure 14: Transient response of D-FF Q output

WinSPICE has better match in digital circuits compared to analog circuits due to the saturation nature of MOS in digital operation; whereas analog circuits often operate in the sub-threshold regime, both simulators do not track analog as close as digital circuits.

\section{Discussion}

FreeVLSI has shown comparable performance with commercial products in SPICE simulation in terms of DC, AC and transient analysis. It is superior judging from cost factor. However, unlike commercial CAD software, FreeVLSI does not have good integrity to interface between design tools. It also lacks a user friendly graphical interface. For users to maneuver smoothly within FreeVLSI framework, some knowledge of computer script is necessary in order to convert and transfer output between different tools.

FreeVLSI also suffers from the simple DRC checking tool. For comprehensive layout, LASI often overlooks DRC errors and has slow loading speed. The next work will look at this issue closely.

\section{CONCLUSION}

In conclusion, these results prove that FreeVLSI can be used as an alternative over commercial CAD tools for simple mixed signal custom circuits, with good matching to actual silicon results. The final design is foundry compatible and can be fabricated using Silterra's University Cooperation program.

The advantage of this framework is its zero cost. In fact FreeVLSI can be installed on low end PCs. This allows more local universities to venture into the world of VLSI design. It is hoped that with the introduction of this framework, more local engineers with VLSI design experience could flourish.

\section{REFERENCES}

[1] http://www.mosis.org

[2] http:/www.europractice.imec.be/europractice /europractice.htm1

[3] http://www.5spice.com

[4] http://www.linear.com/company/software.jsp

[5] http://wwwdevice.eecs.berkeley.edu/ bsim3/ intro.html

[6] http://www.winspice.com

[7] http://members.aol.com/lasicad/

[8] Banba, H. Shiga, T.Miyaba, T.Tanzawa, S.Atsumi, and K.Sakui, "A CMOS Bandgap Reference Circuit with Sub-1-V Operation", IEEE Journal of Solid State Circuits, Vol 34-5, pp 670-674, May 1999. 